\title{
Clinically significant bleeding with low-dose rivaroxaban versus aspirin, in addition to P2Y12 inhibition, in acute coronary syndromes (GEMINI-ACS-1): a double-blind, multicentre, randomised trial
}

\author{
E Magnus Ohman, Matthew T Roe, P Gabriel Steg, Stefan KJames, Thomas J Povsic, Jennifer White, Frank Rockhold, Alexei Plotnikov, \\ Hardi Mundl, John Strony, Xiang Sun, Steen Husted*, Michal Tendera, Gilles Montalescot, M Cecilia Bahit, Diego Ardissino, Héctor Bueno, \\ MarcJ Claeys, Jose C Nicolau, Jan H Cornel, Shinya Goto, Róbert Gábor Kiss, Ümit Güray, Duk-Woo Park, Christoph Bode, Robert C Welsh, \\ CMichael Gibson
}

\section{Summary}

Background Dual antiplatelet therapy (DAPT), aspirin plus a P2Y12 inhibitor, is the standard antithrombotic treatment following acute coronary syndromes. The factor Xa inhibitor rivaroxaban reduced mortality and ischaemic events when added to DAPT, but caused increased bleeding. The safety of a dual pathway antithrombotic therapy approach combining low-dose rivaroxaban (in place of aspirin) with a P2Y12 inhibitor has not been assesssed in acute coronary syndromes. We aimed to assess rivaroxaban $2.5 \mathrm{mg}$ twice daily versus aspirin $100 \mathrm{mg}$ daily, in addition to clopidogrel or ticagrelor (chosen at investigator discretion before randomisation), for patients with acute coronary syndromes started within 10 days after presentation and continued for 6-12 months.

Methods In this double-blind, multicentre, randomised trial (GEMINI-ACS-1) done at 371 clinical centres in 21 countries, eligible patients were older than 18 years with unstable angina, non-ST segment elevation myocardial infarction (NSTEMI) or ST segment elevation myocardial infarction (STEMI), with positive cardiac biomarkers and either ischaemic electrocardiographic changes or an atherosclerotic culprit lesion identified during angiography. Participants were randomly assigned (1:1) within 10 days after admission for the index acute coronary syndromes event to either aspirin or rivaroxaban based on a computer-generated randomisation schedule. Randomisation was balanced by using randomly permuted blocks with size of four and was stratified based on the background P2Y12 inhibitor (clopidogrel or ticagrelor) intended to be used at the time of randomisation. Investigators and patients were masked to treatment assignment. Patients received a minimum of 180 days of double-blind treatment with rivaroxaban $2.5 \mathrm{mg}$ twice daily or aspirin $100 \mathrm{mg}$ daily. The choice of clopidogrel or ticagrelor during trial conduct was not randomised and was based on investigator preference. The primary endpoint was thrombolysis in myocardial infarction (TIMI) clinically significant bleeding not related to coronary artery bypass grafting (CABG; major, minor, or requiring medical attention) up to day 390 . Primary analysis was by intention to treat. This study is registered with ClinicalTrials.gov, number NCT02293395.

Findings Between April 22, 2015, and Oct 14, 2016, 3037 patients with acute coronary syndromes were randomly assigned; 1518 to receive aspirin and 1519 to receive rivaroxaban. 1704 patients (56\%) were in the ticagrelor and $1333(44 \%)$ in the clopidogrel strata. Median duration of treatment was 291 days (IQR 239-354). TIMI non-CABG clinically significant bleeding was similar with rivaroxaban versus aspirin therapy (total 154 patients [5\%]; 80 participants [5\%] of 1519 vs 74 participants [5\%] of 1518; HR 1.09 [95\% CI 0 - 80-1.50]; p=0 5840).

Interpretation A dual pathway antithrombotic therapy approach combining low-dose rivaroxaban with a P2Y12 inhibitor for the treatment of patients with acute coronary syndromes had similar risk of clinically significant bleeding as aspirin and a P2Y12 inhibitor. A larger, adequately powered trial would be required to definitively assess the efficacy and safety of this approach.

Funding Janssen Research \& Development and Bayer AG.

\section{Introduction}

Aspirin has been a foundational therapy for the treatment of acute coronary syndromes for more than 30 years since pivotal trials comparing aspirin versus placebo showed significant reductions in the short-term risk of myocardial infarction and mortality, but few trials have evaluated the efficacy of aspirin as a long-term therapy., ${ }^{1,2}$
Clopidogrel was later added to aspirin (dual antiplatelet therapy [DAPT]) for the treatment of acute coronary syndromes and reduced the risk of the composite outcome of cardiovascular death, myocardial infarction, and stroke for up to 12 months but increased the risk of major bleeding. ${ }^{3}$ Subsequent trials of DAPT comparing more potent P2Y12 inhibitors (prasugrel and ticagrelor)
Published Online March 18, 2017 http://dx.doi.org/10.1016 S0140-6736(17)30751-1 See Online/Comment http://dx.doi.org/10.1016/ S0140-6736(17)30760-2 *In memoriam of Dr Steen Husted Duke Clinical Research Institute, Duke University School of Medicine, Durham, NC, USA (Prof E M Ohman MB, Prof MT Roe MD, T J Povsic MD JWhite MS,

Prof F Rockhold PhD); DHU FIRE, Université Paris-Diderot AP-HP and Inserm U-1148, Paris, France (Prof P G Steg MD) and NHLI Royal Brompton Hospital, Imperial College London, London, UK (Prof P G Steg); Department of Medical Sciences and Uppsala Clinical Research Center, Uppsala University, Uppsala, Sweden (Prof S K James MD); Janssen Research and Development, Raritan, NJ, USA (A Plotnikov MD, J Strony MD $X$ Sun PhD); Bayer AG, Wuppertal, Germany (H Mundl MD); Department of Cardiology, Aarhus University Hospital, Aarhus, Denmark (Prof S Husted MD); 3rd Division of Cardiology, Medical University of Silesia, Katowice Poland (Prof M Tendera MD); Institut de Cardiologie, Groupe Hospitalier Pitié-Salpêtrière, Paris, France (Prof G Montalescot MD); INECO Neurociencias Oroño, Rosario, Santa Fe, Argentina (MC Bahit MD); Divisione di Cardiologia, Policlinico San Matteo, Pavia, Italy (Prof D Ardissino MD); Spanish National Centre for Cardiovascular Research, Madrid, Spain 
(Prof H Bueno MD); University Hospital Antwerp, Edegem, Belgium (Prof M J Claeys MD); Heart Institute (InCor), University of São Paulo Medical School, São Paulo, Brazil (Prof J C Nicolau MD); Department of Cardiology, Noordwest Ziekenhuisgroep, Alkmaar and Dutch Network for Cardiovascular Research (WCN) Netherlands (J H Cornel MD); Department of Medicine (Cardiology), Tokai University School of Medicine, Isehara, Japan (Prof S Goto MD); Department of Cardiology, Military Hospital, Budapest, Hungary (Prof R G Kiss MD); Numune Education and Research Hospital, Department of Cardiology, Ankara, Turkey (Ü Güray MD); Division of Cardiology, Asan Medical Center, University of Ulsan College of Medicine, Seoul, Korea (D-W Park MD); University of Freiburg, Freiburg, Germany (Prof C Bode MD); Mazankowski

Alberta Heart Institute and University of Alberta, Edmonton, AB, Canada (Prof R CWelsh MD); and PERFUSE Study Group, Beth Israel Deaconess Hospital, Harvard Medical School, Boston, MA, USA (Prof C M Gibson MD)

Correspondence to: Dr E Magnus Ohman, Duke University Medical Center, 8676A HAFS Building, Box 3126 DUMC, Durham, NC 27710, USA

ohman001@mc.duke.edu
Research in context

\section{Evidence before this study}

We searched MEDLINE, Embase, and the Cochrane Register of Controlled Trials from inception to Dec 31, 2016, using the following keywords: myocardial infarction, acute coronary syndrome, unstable angina, anticoagulants, purinergic $\mathrm{P} 2 \mathrm{Y}$ receptor antagonists, platelet aggregation inhibitors, antiplatelets, apixaban, rivaroxaban, aspirin, clopidogrel, prasugrel, ticlopidine, or ticagrelor, for English language articles only. A review of references from appropriate articles was done to identify additional studies. After reviewing the full text of these studies, we concluded that no trial has explored the use of a factor Xa inhibitor plus a P2Y12 inhibitor without aspirin.

\section{Added value of this study}

To the best of our knowledge, GEMINI-ACS-1, for the first time, showed that low-dose rivaroxaban, when used with either ticagrelor or clopidogrel, did not increase clinically significant or

with clopidogrel showed further reductions in composite ischaemic events, but also an increased risk of major bleeding. 4.5 Although the ischaemic findings from these trials of DAPT have led to consistent guideline recommendations for DAPT for post-acute coronary syndromes therapy, ${ }^{6-9}$ nearly $10 \%$ of patients treated with optimal DAPT therapy still suffer major cardiovascular events during follow-up..$^{10}$

Subsequent trials of post-acute coronary syndromes have explored triple antithrombotic therapy, adding an oral anticoagulant to DAPT with aspirin plus clopidogrel. ${ }^{11,12}$ Although low-dose rivaroxaban reduced the composite endpoint of cardiovascular death, myocardial infarction, and stroke, ${ }^{11}$ no benefits were noted with standard-dose apixaban in this setting, ${ }^{12}$ and both studies showed a significant three-fold to four-fold increase in major bleeding, suggesting that a ceiling might have been reached for adding antithrombotic therapies in the post-acute coronary syndromes setting. Notwithstanding these observations, recent trials assessing patients with an indication for full-dose oral anticoagulation undergoing percutaneous coronary intervention (PCI), where triple antithrombotic therapy (aspirin plus clopidogrel together with standard doses of oral anticoagulants) has been the standard of care, have suggested that withholding aspirin has not caused a significant increase in ischaemic events and showed a lower risk of major bleeding events. ${ }^{13,14}$ Recent in-vivo thrombosis and bleeding studies have assessed rivaroxaban in combination with $\mathrm{P} 2 \mathrm{Y} 12$ inhibitors and found this combination to have similar efficacy as DAPT, but with a lower risk of bleeding. ${ }^{15,16}$ These findings suggest that the role of a dual pathway antithrombotic strategy using an oral anticoagulant (in place of aspirin) with a P2Y12 inhibitor warrants additional exploration in the post-acute coronary syndromes setting. major bleeding compared with dual antiplatelet therapy, establishing this strategy as a safe approach for post-acute coronary syndromes treatment. Furthermore, there was no signal of an increased risk of stent thrombosis after cessation of aspirin therapy.

\section{Implications of all the available evidence}

Our trial suggests that substituting aspirin with low-dose rivaroxaban is not associated with an appreciable increase in bleeding and appears safe; however, GEMINI-ACS-1 was not powered to assess effect on ischaemic events. Whether such a strategy would be associated with a net clinical benefit and improved outcomes, in particular a lower risk of mortality or composite ischaemic events post-ACS, would require a definitive cardiovascular outcomes trial. In view of the risk of recurrent events in this patient population, additional study of this approach seems warranted.

We explored the use of a low dose of the oral anticoagulant rivaroxaban, a potent direct factor $\mathrm{Xa}$ inhibitor, in place of aspirin, together with a P2Y12 inhibitor (clopidogrel or ticagrelor) in the post-acute coronary syndromes setting. ${ }^{17}$ We aimed to investigate the safety of this novel dual pathway antithrombotic regimen using clinically significant bleeding as a measure of antithrombotic therapy potency. ${ }^{18,19}$

\section{Methods}

\section{Study design and participants}

The study has been described in detail elsewhere. ${ }^{17}$ In brief, the GEMINI-ACS-1 trial, was a phase 2, multicentre, double-blind randomised trial, done between April 22, 2015, and Oct 14, 2016, at 321 clinical centres in 21 countries. Eligible patients were older than 18 years with unstable angina, non-ST segment elevation myocardial infarction (NSTEMI), or ST segment elevation myocardial infarction (STEMI). All patients with STEMI and NSTEMI required positive cardiac biomarkers and either ischaemic electrocardiographic changes or an atherosclerotic culprit lesion identified during angiography. Additional inclusion criteria were a history of diabetes or previous myocardial infarction for patients younger than 55 years; and, for patients with unstable angina, at least one of the following: ischaemic electrocardiographic changes, revascularisation for the index acute coronary syndromes event, or a thrombolysis in myocardial infarction (TIMI) risk score of 4 or more. Major exclusion criteria were history of active bleeding, intracranial haemorrhage, or clinically significant gastrointestinal bleeding within 12 months. Other exclusion criteria were creatinine clearance less than $20 \mathrm{~mL} / \mathrm{min}$, use of omeprazole for patients in the clopidogrel stratum, and need for chronic full-dose oral anticoagulation..$^{17}$ 
The study was approved by local institutional review boards. All patients gave written informed consent. Central randomisation was implemented in this study.

\section{Randomisation and masking}

Patients were randomly assigned (1:1) to either aspirin or rivaroxaban based on a computer-generated randomisation schedule. Randomisation was balanced by using randomly permuted blocks with size of four and was stratified based on the background P2Y12 inhibitor (clopidogrel or ticagrelor) intended to be used at the time of randomisation. The choice of clopidogrel or ticagrelor during trial conduct was not randomised and was based on investigator preference and country availability (ticagrelor was not approved in Japan during the trial). The interactive web response system assigned a unique treatment code, which dictated treatment assignment for the patient. Investigators and patients were masked to treatment assignment. The study drugs (ie, rivaroxaban and rivaroxaban placebo, aspirin and aspirin placebo) were identical in appearance and were packaged in identical containers.

\section{Procedures}

Patients had to be on a stable ( $>48$ hours) dose of clopidogrel (75 mg daily) or ticagrelor $(90 \mathrm{mg}$ twice daily) plus aspirin by the time of randomisation. Generic and branded clopidogrel and branded ticagrelor were supplied, based on national availability, to patients during their participation in the study to minimise bias and late crossover. Genetic testing for clopidogrel poormetaboliser status was done centrally in all patients, with results available to investigators 1-2 weeks after randomisation. Patients received low-dose rivaroxaban (2.5 mg twice daily) plus a P2Y12 inhibitor (clopidogrel or ticagrelor) or aspirin (100 mg daily) plus a P2Y12 inhibitor (clopidogrel or ticagrelor). Patients were seen for study visits at 30,90,180, and 270 days and at the end of treatment (up to 30 days after a maximum of 360 days of therapy). A minimum of 180 days of double-blind treatment with rivaroxaban $2 \cdot 5 \mathrm{mg}$ twice daily versus aspirin $100 \mathrm{mg}$ daily was required. The proportion of STEMI patients was capped at $50 \%$ so as to achieve a balanced acute coronary syndromes population.

\section{Outcomes}

The primary endpoint was TIMI non-coronary artery bypass graft (CABG) surgery clinically significant bleeding (non-CABG major, minor, or requiring medical attention) up to day 390. Other bleeding measures included Global Use of Strategies to Open Occluded Coronary Arteries (GUSTO), Bleeding Academic Research Consortium (BARC), and International Society on Thrombosis and Haemostasis (ISTH) bleeding criteria. Exploratory ischaemic endpoints included the composite of cardiovascular death, myocardial infarction, stroke, or definite stent thrombosis; all-cause death; and individual component ischaemic endpoints measured throughout the entire study period (intention-to-treat population). All bleeding and ischaemic events were independently adjudicated by a clinical events committee blinded to treatment assignment using previously published criteria. ${ }^{20-22} \mathrm{~A}$ data safety monitoring committee reviewed unblinded data throughout the trial.

\section{Statistical analysis}

GEMINI-ACS- 1 was designed as a phase 2 trial to estimate the bleeding risk of rivaroxaban compared with aspirin on top of standard P2Y12 inhibitor therapy with either clopidogrel or ticagrelor. ${ }^{17}$ The estimated TIMI clinically significant bleeding rate was based on results from the ATLAS ACS-2 trial with rivaroxaban $2.5 \mathrm{mg}$ twice daily using similar inclusion criteria in the post-acute coronary syndromes setting. ${ }^{11}$ The expected bleeding rate in the DAPT group (aspirin and clopidogrel) was $4.5 \%$ at 180 days and $6.5 \%$ at 360 days with an estimated $10 \%$ discontinuation rate. The limit of sensitivity for an upper bound of $2 \cdot 0$ was chosen based on the observation that most trials assessing triple antithrombotic therapies have noted a greater than two-fold increase in the risk of bleeding. The following assumptions were used to derive the sample size of the trial. An observed hazard ratio (HR) of 1.0 between rivaroxaban and aspirin would result in an upper bound of a two-sided 95\% CI of 1.4 with 170 total bleeding events, while an observed HR of 1.5 for bleeding would result in an upper bound of a two-sided $95 \%$ CI of $2 \cdot 0$. Corresponding $95 \%$ CI upper bounds for each stratum (clopidogrel or ticagrelor) with 85 bleeding events for an observed HR of 1.0 is $1 \cdot 5$, while an observed HR of $1 \cdot 3$ would have an upper bound of $2 \cdot 0$. Using prior estimates for bleeding rates at 6 months and 12 months from the ATLAS ACS-2 trial, roughly 3000 patients (1500 in each stratum) would be needed for enrolment to provide some confidence in the bleeding effect of a dual pathway strategy compared with DAPT therapy. ${ }^{11}$

The primary analysis was done in the intention-to-treat population using a Cox proportional hazards model from time of randomisation to the first TIMI non-CABG clinically significant bleeding event. Patients were analysed according to randomised treatment group, regardless of actual treatment received. A modified intention-to-treat analysis was used for bleeding events and included from time of randomisation to 2 days after last dose of study drug. For patients who did not have events, censoring was done at the date of last dose of study drug plus 2 days or the last clinical evaluation date (whichever came first).

Homogeneity of treatment effects across prespecified subgroups was explored with a test for interaction using Cox proportional hazards models. Each P2Y12 inhibitor stratum was also analysed using similar methods as for the primary endpoint. Exploratory analyses of ischaemic endpoints, including the composite of cardiovascular death, myocardial infarction, stroke, or definite stent 
thrombosis; all-cause death; and individual component ischaemic endpoints were done using Kaplan-Meier estimates and Cox proportional models in the intentionto-treat population throughout the study period. A p value less than 0.05 was considered statistically significant. Several analyses were done after the database was locked and should therefore be regarded as hypothesisgenerating only. A landmark analysis set at 30 days was done on both the primary TIMI non-CABG clinically significant bleeding and ischaemic composite outcomes and is shown in the appendix ( $\mathrm{p}$ 13). A Cox proportional hazards regression multivariate analysis was done to assess the choice of P2Y12 inhibitor on both the primary bleeding endpoint and the ischaemic composite outcome and is shown in the appendix (p 9). Adjustments were made for age, race, sex, qualifying event diagnosis, region, randomised treatment, and P2Y12 inhibitor use at the time of randomisation only due to the limited number of endpoints. Finally, a non-inferiority analysis was also done for the primary bleeding endpoint using the upper $95 \%$ bound of the hazard ratio at $2 \cdot 0$. Data were analysed with SAS software, version 9.3 or higher (SAS Institute, Inc., Cary, NC, USA).

This study is registered with ClinicalTrials.gov, number NCT02293395.

\section{Role of the funding source}

Janssen Research \& Development (Raritan, NJ, USA) and Bayer AG (Wuppertal, Germany) funded the GEMINI-ACS-1 trial and supported this analysis through a grant to Duke University and Harvard School of Medicine. All analyses were done at the Duke Clinical

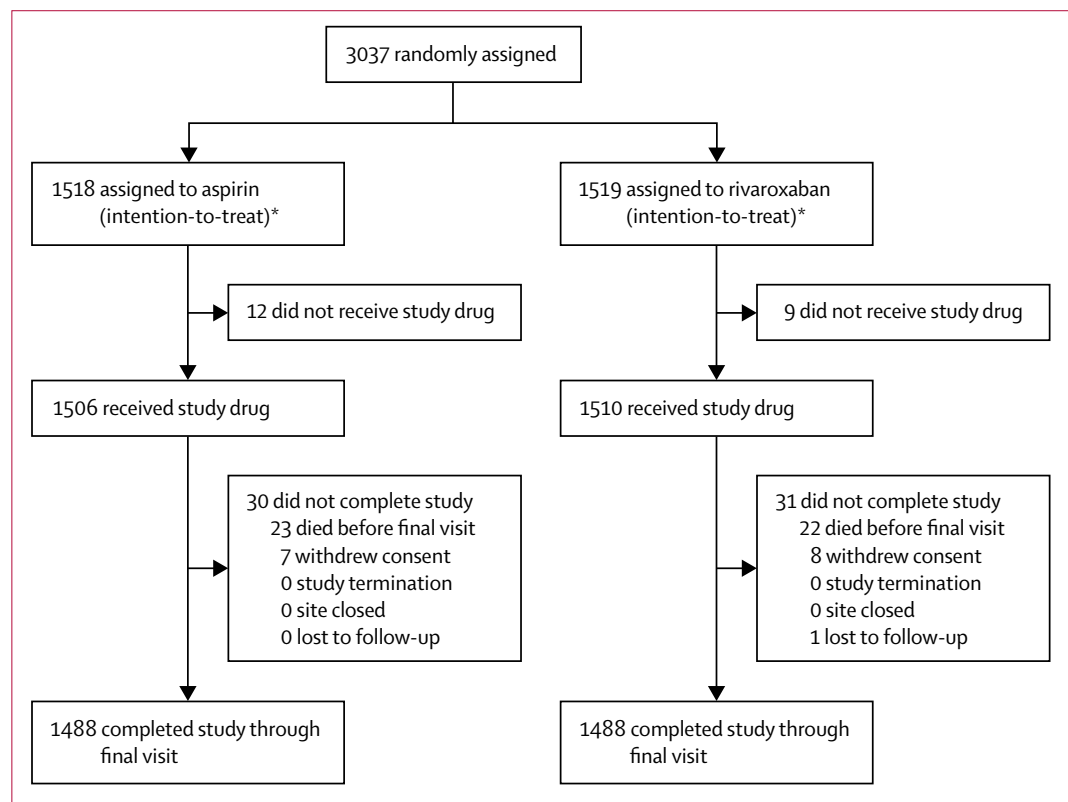

Figure 1: Trial profile

${ }^{*}$ A modified intention-to-treat analysis was used for bleeding events and included from time of randomisation to 2 days after last dose of study drug.
Research Institute (Durham, NC, USA) and all authors had full access to the data. The Duke Clinical Research Institute coordinated the trial, managed the database, and did the secondary and post-hoc analyses for this report, independent of the sponsors. An international executive committee designed the trial and was responsible for oversight of study conduct and reporting of all results and takes responsibility for the accuracy and completeness of data analyses. The authors are fully responsible for the study design, data collection, analysis and interpretation of the data, and writing of the report. All authors agreed to submit the report for publication; the funder had no role in this decision.

\section{Results}

Between April 22, 2015 and Oct 14, 2016, 3037 patients with acute coronary syndromes were randomly assigned; 1518 to receive aspirin and 1519 to receive rivaroxaban at a median of 5.5 days (IQR 3.4-7.6; figure 1) after the index acute coronary syndromes event; bleeding events were censored 3 days after randomisation for participants who did not receive study drug (figure 1). Only one patient was lost to follow-up during the study period (figure 1). At randomisation, 1704 patients were treated with ticagrelor and 1333 were treated with clopidogrel. Median duration of treatment with blinded study drug was 291 days (IQR 239-354) and median duration of follow-up was 326 days (284-383). Premature treatment discontinuation (before the final study visit) occurred in $172(11 \%)$ patients treated with rivaroxaban versus 193 (13\%) treated with aspirin. During study conduct, 197 (6\%) of 3037 patients switched between clopidogrel and ticagrelor (from the original P2Y12 inhibitor chosen and prespecified before randomisation). Discontinuation of P2Y12 inhibitor therapy (before the final study visit) was $4.4 \%$ for the overall population.

Baseline characteristics of the population represent a typical post-acute coronary syndromes population with an average age of 63 years and a high prevalence of risk factors (table 1). Baseline demographics were balanced between the two treatment groups. Of the overall population, 1484 (49\%) presented with STEMI, 1223 (40\%) with NSTEMI, and 330 (11\%) with unstable angina. Most patients (2855 [94\%]) had cardiac catheterisation and $2645(87 \%)$ were treated with PCI for their index acute coronary syndromes event before randomisation. Patients were predominantly enrolled from Europe (2331 [77\%]). Baseline characteristics among patients treated with ticagrelor or clopidogrel are shown in the appendix (p 4); patients treated with ticagrelor were younger; less likely to have diabetes, previous myocardial infarction, previous PCI; more likely to undergo PCI for the index acute coronary syndromes event, and more likely to be enrolled from North America and western Europe.

The primary endpoint of TIMI non-CABG clinically significant bleeding was noted in 154 patients (5\%; 80 participants [5\%] in the rivaroxaban group and 74 [5\%] 
in the aspirin group; HR $1 \cdot 09,95 \%$ CI $0 \cdot 80-1 \cdot 50$; $\mathrm{p}=0 \cdot 5840$; table 2 ; figure 2 ). The most common type of bleeding was TIMI bleeding requiring medical attention (62 patients [4\%] of 1519 in the rivaroxaban group and 62 [4\%] of 1518 in the aspirin group), with a low frequency of severe or major bleeding events using TIMI bleeding definitions. Using the ISTH definition for major bleeding, a higher rate of bleeding was noted with rivaroxaban (31 [2\%]) than with aspirin (17 [1\%]; $\mathrm{p}=0 \cdot 0420$ ), but there were no differences using GUSTO or BARC bleeding definitions, regardless of severity (table 2).

No significant interaction was noted between any of the subgroups examined and treatment assignment (rivaroxaban $v$ s aspirin) with regard to clinically significant bleeding (figure 3). The frequency of TIMI non-CABG clinically significant bleeding for rivaroxaban versus aspirin was similar in the ticagrelor stratum (58 [7\%] vs 51 [6\%]; HR 1.15 [95\% CI 0.79-1.68]) compared with the clopidogrel stratum (22 [3\%] vs 23 [3\%], 0.95 [0.53-1.71]) with no significant treatment interactions noted $(\mathrm{p}=0 \cdot 5889$; appendix $\mathrm{p} 6$ ).

For the exploratory ischaemic endpoint, the frequency of the composite ischaemic endpoint of cardiovascular death, myocardial infarction, stroke, or definite stent thrombosis was 76 participants (5\%) in the rivaroxaban group versus 72 (5\%) with aspirin (HR 1.06 [95\% CI $0.77-1.46]$; $\mathrm{p}=0 \cdot 7316$; table 3 , figure 4 ). The frequency of all-cause death and the component ischaemic endpoints were also similar by treatment assignment (table 3). Appendix ( $p 12$ ) shows the Kaplan-Meier curves for the composite ischaemic endpoint by treatment assignment among those treated with ticagrelor versus clopidogrel. No significant interaction was noted by treatment assignment for the P2Y12 inhibitor strata $(\mathrm{p}=0.3889$; appendix $\mathrm{p}$ 8). Frequencies of the composite ischaemic endpoint and individual component ischaemic endpoints by rivaroxaban versus aspirin treatment assignment among those treated with ticagrelor versus clopidogrel are shown in the appendix (p 9).

For the exploratory post-hoc analysis, a landmark analysis was done for the primary endpoint of TIMI nonCABG clinically significant bleeding and showed a numerically higher number of events in the first 30 days among patients randomly assigned to rivaroxaban versus aspirin (appendix p 13). However, no significant difference $(\mathrm{p}=0 \cdot 149)$ was noted in the number of events in the first 30 days, but the curves were superimposed from 30 days and onward. Similarly, a landmark analysis of the ischaemic composite outcome showed a numerically higher number of events in the first 30 days with superimposable event rates after, but this difference was also not significant ( $\mathrm{p}=0 \cdot 211$; appendix $\mathrm{p} 13)$.

The relative effect of either clopidogrel or ticagrelor on the primary bleeding endpoint was assessed using a multivariable model with adjustments for age, sex, race, qualifying event diagnosis, region, and study treatment

\begin{tabular}{|c|c|c|c|}
\hline & Aspirin ( $n=1518)$ & Rivaroxaban (n=1519) & Total $(n=3037)$ \\
\hline \multicolumn{4}{|l|}{ Demographics } \\
\hline Age (years) & $63(57-69)$ & $62(57-69)$ & $62(57-69)$ \\
\hline Age $\geq 65$ years & $629(41 \%)$ & $632(42 \%)$ & $1261(42 \%)$ \\
\hline Weight (kg) & $80 \cdot 0(72 \cdot 0-91 \cdot 3)$ & $81 \cdot 2(71 \cdot 3-92 \cdot 0)$ & $80.6(71 \cdot 6-92 \cdot 0)$ \\
\hline Weight $<60 \mathrm{~kg}$ & $82(5 \%)$ & $79(5 \%)$ & $161(5 \%)$ \\
\hline Men & $1141(75 \%)$ & 1134 (75\%) & $2275(75 \%)$ \\
\hline White race & $1407(93 \%)$ & $1417(93 \%)$ & $2824(93 \%)$ \\
\hline \multicolumn{4}{|l|}{ Presentation characteristics } \\
\hline \multicolumn{4}{|l|}{ Disease classification } \\
\hline STEMI & $741(49 \%)$ & $743(49 \%)$ & $1484(49 \%)$ \\
\hline NSTEMI & $612(40 \%)$ & $611(40 \%)$ & $1223(40 \%)$ \\
\hline Unstable angina & $165(11 \%)$ & $165(11 \%)$ & $330(11 \%)$ \\
\hline $\begin{array}{l}\text { Time from hospital admission } \\
\text { to randomisation (days) }\end{array}$ & $5 \cdot 1(3 \cdot 0-7 \cdot 3)$ & $5 \cdot 1(3 \cdot 1-7 \cdot 2)$ & $5 \cdot 1(3 \cdot 1-7 \cdot 2)$ \\
\hline \multicolumn{4}{|l|}{ Cardiovascular risk factors } \\
\hline Hypertension & 1139 (75\%) & $1085(71 \%)$ & 2224 (73\%) \\
\hline Hyperlipidaemia & $854(56 \%)$ & $855(56 \%)$ & $1709(56 \%)$ \\
\hline Diabetes & $460(30 \%)$ & $446(29 \%)$ & $906(30 \%)$ \\
\hline Current smoker & $511(34 \%)$ & $486(32 \%)$ & 997 (33\%) \\
\hline \multicolumn{4}{|l|}{ Cardiovascular disease history } \\
\hline Previous myocardial infarction & $345(23 \%)$ & $314(21 \%)$ & $659(22 \%)$ \\
\hline Previous $\mathrm{PCl}$ & $315(21 \%)$ & $286(19 \%)$ & $601(20 \%)$ \\
\hline Previous CABG & $68(4 \%)$ & $58(4 \%)$ & $126(4 \%)$ \\
\hline Previous PAD & $74(5 \%)$ & $66(4 \%)$ & $140(5 \%)$ \\
\hline Previous heart failure & $153(10 \%)$ & $157(10 \%)$ & $310(10 \%)$ \\
\hline \multicolumn{4}{|l|}{ Baseline risk assessment } \\
\hline GRACE risk score & $97 \cdot 0(83 \cdot 0-112 \cdot 0)$ & $96 \cdot 0(83 \cdot 0-112 \cdot 0)$ & $96 \cdot 0(83 \cdot 0-112 \cdot 0)$ \\
\hline Haemoglobin (g/dL) & $14 \cdot 1(13 \cdot 1-15 \cdot 1)$ & $14 \cdot 2(13 \cdot 2-15 \cdot 1)$ & $14 \cdot 1(13 \cdot 1-15 \cdot 1)$ \\
\hline Creatinine clearance (mL/min) & $87 \cdot 0(70 \cdot 2-106 \cdot 2)$ & $87 \cdot 0(69 \cdot 0-106 \cdot 8)$ & $87 \cdot 0(69 \cdot 6-106 \cdot 8)$ \\
\hline \multicolumn{4}{|c|}{ Cardiac procedures for index event } \\
\hline Catheterisation done & $1430(94 \%)$ & $1425(94 \%)$ & $2855(94 \%)$ \\
\hline $\mathrm{PCl}$ done & $1320(87 \%)$ & $1325(87 \%)$ & $2645(87 \%)$ \\
\hline Stent placed & $1286(85 \%)$ & $1295(85 \%)$ & $2581(85 \%)$ \\
\hline DES & $870(68 \%)$ & $859(66 \cdot 5 \%)$ & $1729(67 \%)$ \\
\hline BMS & $423(33 \%)$ & $438(34 \%)$ & $861(33 \cdot 5 \%)$ \\
\hline Bioabsorbable stent & $8(1 \%)$ & $16(1 \%)$ & $24(1 \%)$ \\
\hline CABG performed & $4(<1 \%)$ & $5(<1 \%)$ & $9(<1 \%)$ \\
\hline \multicolumn{4}{|l|}{ Geographical region } \\
\hline North America & $135(9 \%)$ & $130(9 \%)$ & $265(9 \%)$ \\
\hline South America & $150(10 \%)$ & $143(9 \%)$ & $293(10 \%)$ \\
\hline Western Europe & $292(19 \%)$ & $326(21 \%)$ & $618(20 \%)$ \\
\hline Eastern Europe & $373(25 \%)$ & $374(25 \%)$ & $747(25 \%)$ \\
\hline Central Europe & $488(32 \%)$ & $478(31 \%)$ & $966(32 \%)$ \\
\hline Asia and Pacific & $80(5 \%)$ & $68(4 \%)$ & $148(5 \%)$ \\
\hline \multicolumn{4}{|c|}{ Concomitant medication at randomisation } \\
\hline Beta-blocker & $984(65 \%)$ & $970(64 \%)$ & $1954(64 \%)$ \\
\hline ACE inhibitors/ARB & $960(63 \%)$ & $947(62 \%)$ & $1907(63 \%)$ \\
\hline Statins & $1065(70 \%)$ & $1038(68 \%)$ & $2103(69 \%)$ \\
\hline \multicolumn{4}{|c|}{$\begin{array}{l}\text { Data are } \mathrm{n}(\%) \text { or median (IQR). STEMI=ST segment elevation myocardial infarction. NSTEMI=non-ST segment } \\
\text { elevation myocardial infarction. } \mathrm{PCl}=\text { percutaneous coronary intervention. } \mathrm{CABG}=\text { coronary artery bypass graft. } \\
\mathrm{PAD}=\text { peripheral artery disease. GRACE=Global Registry of Acute Coronary Events. } \mathrm{DES}=\text { =drug-eluting stent. } \\
\mathrm{BMS}=\text { bare-metal stent. } \mathrm{ACE}=\text { angiotensin converting enzyme. } \mathrm{ARB}=\text { angiotensin receptor blocker. }\end{array}$} \\
\hline
\end{tabular}




\begin{tabular}{|c|c|c|c|c|}
\hline & $\begin{array}{l}\text { Aspirin } \\
(n=1518)\end{array}$ & $\begin{array}{l}\text { Rivaroxaban } \\
(\mathrm{n}=1519)\end{array}$ & $\begin{array}{l}\mathrm{HR} \\
(95 \% \mathrm{Cl})\end{array}$ & $p$ value \\
\hline \multicolumn{5}{|l|}{ TIMI bleeding categories } \\
\hline $\begin{array}{l}\text { TIMI non-CABG clinically } \\
\text { significant bleeding }\end{array}$ & $74(5 \%)$ & $80(5 \%)$ & $1.09(0.80-1.50)$ & 0.5840 \\
\hline TIMI fatal bleeding & 0 & $2(<1 \%)$ & NA & NA \\
\hline Intracranial haemorrhage & 0 & $1(<1 \%)$ & NA & NA \\
\hline TIMI major bleeding* & $8(1 \%)$ & $10(1 \%)$ & $1.25(0 \cdot 49-3 \cdot 17)$ & 0.6341 \\
\hline TIMI non-CABG major bleeding & $8(1 \%)$ & $10(1 \%)$ & $1.25(0.49-3 \cdot 17)$ & 0.6341 \\
\hline TIMI minor bleeding & $4(<1 \%)$ & $9(1 \%)$ & $2.25(0.69-7.29)$ & 0.1664 \\
\hline $\begin{array}{l}\text { TIMI bleeding requiring medical } \\
\text { attention }\end{array}$ & $62(4 \%)$ & $62(4 \%)$ & $1.01(0.71-1.44)$ & 0.9581 \\
\hline TIMI insignificant bleeding & $25(2 \%)$ & $21(1 \%)$ & $0.84(0.47-1 \cdot 50)$ & 0.5504 \\
\hline \multicolumn{5}{|l|}{ GUSTO bleeding categories } \\
\hline $\begin{array}{l}\text { GUSTO life threatening or severe } \\
\text { bleeding }\end{array}$ & $2(<1 \%)$ & $3(<1 \%)$ & $1.50(0.25-8.95)$ & 0.6571 \\
\hline $\begin{array}{l}\text { GUSTO life threatening, severe, } \\
\text { or moderate bleeding }\end{array}$ & $7(<1 \%)$ & $11(1 \%)$ & $1.58(0.61-4.08)$ & $0 \cdot 3395$ \\
\hline $\begin{array}{l}\text { GUSTO life threatening, severe, } \\
\text { moderate, or mild bleeding }\end{array}$ & $96(6 \%)$ & $99(7 \%)$ & $1.04(0.79-1 \cdot 38)$ & 0.7869 \\
\hline \multicolumn{5}{|l|}{ ISTH bleeding categories } \\
\hline ISTH major bleeding & $17(1 \%)$ & $31(2 \%)$ & $1.83(1.01-3 \cdot 31)$ & 0.0420 \\
\hline \multicolumn{5}{|l|}{ BARC bleeding categories } \\
\hline BARC $3 a$ and higher bleeding & $13(1 \%)$ & $22(1 \%)$ & $1.70(0.85-3 \cdot 37)$ & 0.1263 \\
\hline BARC $3 \mathrm{~b}$ and higher bleeding & $8(0.5 \%)$ & $11(1 \%)$ & $1.38(0.55-3.43)$ & 0.4882 \\
\hline BARC fatal bleeding & 0 & $2(<1 \%)$ & NA & NA \\
\hline \multicolumn{5}{|c|}{$\begin{array}{l}\text { Data are } n \text { (\%), unless otherwise indicated. All bleeding events are non-CABG related with the exception of TIMI maj } \\
\text { bleeding; this category includes both non-CABG and CABG-related TIMI major bleeding. HR=hazard ratio. } \\
\text { TIMI=thrombolysis in myocardial infarction. CABG=coronary artery bypass graft. NA=not applicable. GUSTO=Global } \\
\text { Use of Strategies to Open Occluded Coronary Arteries. ISTH=International Society on Thrombosis and Haemostasis. } \\
\text { BARC=Bleeding Academic Research Consortium. }{ }^{*} T I M I \text { major bleeding is the only endpoint that includes } \\
\text { CABG-related bleeding. }\end{array}$} \\
\hline
\end{tabular}

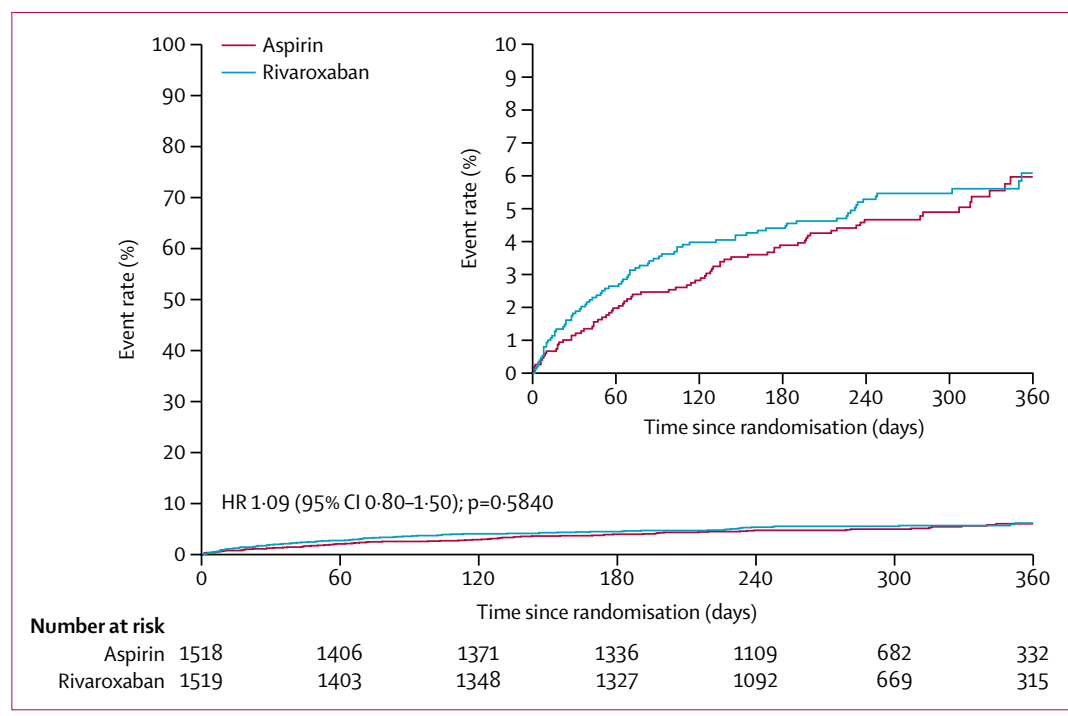

Figure 2: TIMI non-CABG clinically significant bleeding between treatment groups

$\mathrm{TIMI}=$ thrombolysis in myocardial infarction. $\mathrm{CABG}=$ coronary artery bypass graft. $\mathrm{HR}=$ hazard ratio.

(appendix $\mathrm{p}$ 9). In this adjusted analysis, the use of ticagrelor versus clopidogrel was associated with a significant increase in bleeding rate $(\mathrm{p}=0.0006)$, which was also associated with the effect of region on bleeding $(\mathrm{p}=0 \cdot 0178$; appendix $\mathrm{p} 9)$. The randomised treatment assignment (rivaroxaban $v s$ aspirin) was not associated with the bleeding endpoint $(\mathrm{p}=0 \cdot 6152)$, which is consistent with the overall trial results. Although there was a numerically lower rate of the composite ischaemic endpoint with tigagrelor than clopidogrel, this was not statistically significant $(\mathrm{p}=0 \cdot 1487$; appendix $\mathrm{p} 10)$.

\section{Discussion}

The GEMINI-ACS-1 trial sought to assess the safety of substituting a low dose of the oral anticoagulant rivaroxaban for aspirin in patients treated with a P2Y12 inhibitor (either clopidogrel or ticagrelor) in the postacute coronary syndromes setting, when the therapy was started on average 5 days after the index event. We showed a similar risk of TIMI non-CABG clinically significant bleeding with rivaroxaban versus aspirin with consistent results found in prespecified subgroups. Similar results were also noted with other bleeding definitions, including GUSTO and BARC, but increased bleeding rates were reported with the ISTH major bleeding definition. However, interpretation of this single bleeding definition in a post-acute coronary syndromes population should be done with caution in comparison with the results from the other bleeding definitions used. Additionally, no significant interaction was noted for the risk of clinically significant bleeding by P2Y12 inhibitor strata (ticagrelor vs clopidogrel), but these stratified bleeding analyses were exploratory and hypothesis generating.

Several trials have attempted to add an oral anticoagulant to DAPT in the post-acute coronary syndromes setting leveraging a triple antithrombotic therapy approach. Although the ATLAS ACS- 2 trial ${ }^{19}$ with the same low-dose of rivaroxaban studied in GEMINIACS-1 showed a significant reduction in the risk of the composite outcome of cardiovascular death, myocardial infarction, and stroke and in the risk of all-cause death, the rate of major bleeding was three-fold to four-fold higher when rivaroxaban was added to aspirin and clopidogrel in the post-acute coronary syndromes setting. ${ }^{11}$ A similar trial (APPRAISE-2) ${ }^{12}$ assessing the standard dose of apixaban (used for the treatment of atrial fibrillation) plus DAPT in the post-acute coronary syndromes setting was prematurely terminated due to an excessive risk of intracranial haemorrhage and major bleeding with triple antithrombotic therapy, with no difference in the risk of recurrent ischaemic events. ${ }^{12}$ In addition, the WOEST trial ${ }^{13}$ done in the post-PCI setting among patients requiring oral anticoagulation, used a different strategy by removing aspirin from the antithrombotic regimen and showed a significantly lower rate of major bleeding among patients randomly assigned to clopidogrel plus a vitamin $\mathrm{K}$ antagonist oral anticoagulant (at therapeutic doses) than those assigned 


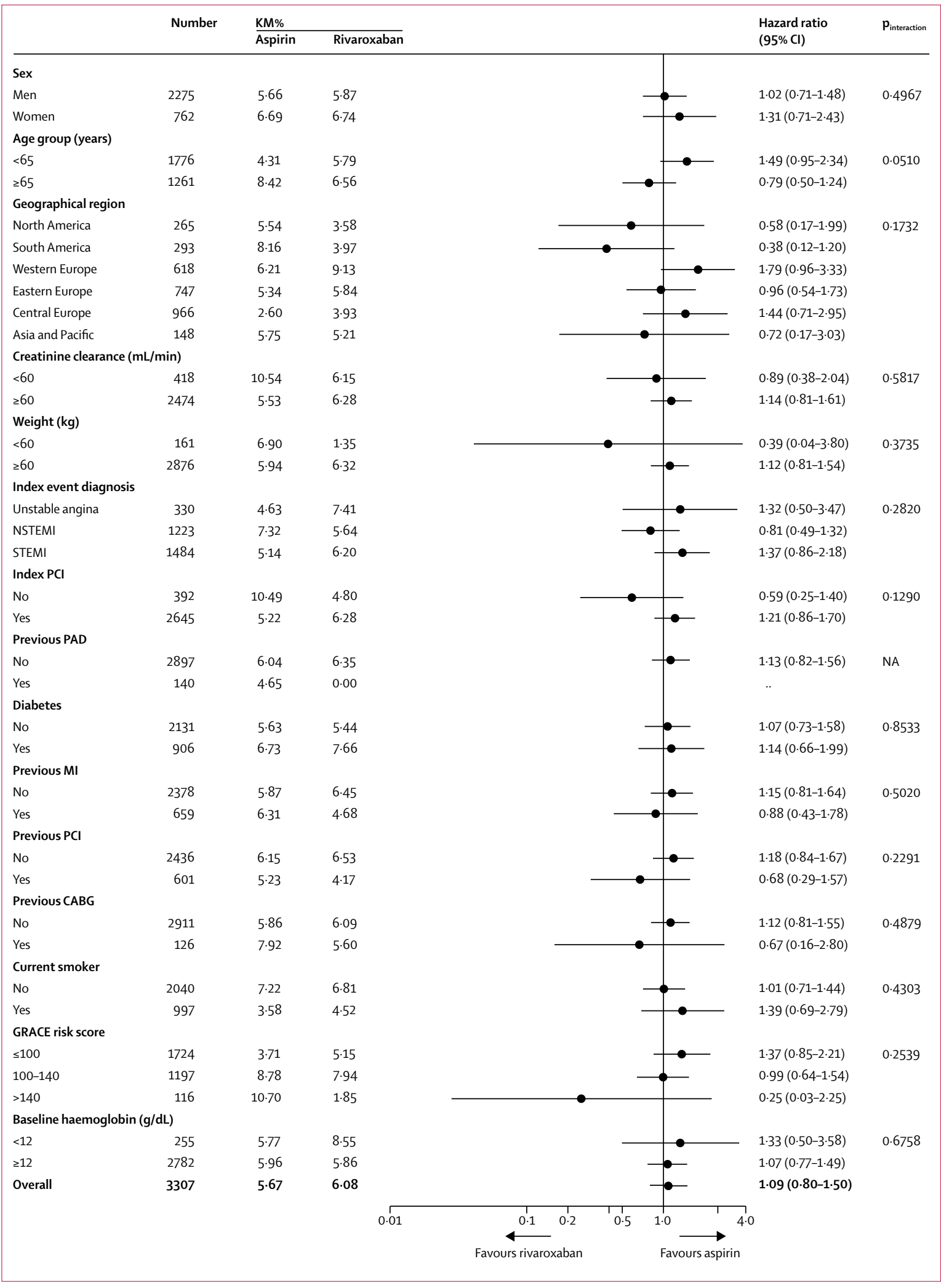

Figure 3: TIMI non-CABC clinically significant bleeding among prespecified subgroups Data include Kaplan-Meier estimates (KM\%) censored at 360 days (region was censored at 330 days). Hazard ratios and 95\% Cls are from Cox proportional hazard models with subgroup, treatment and subgroup by treatment interactions; $p$ values for the interactions. Models were stratified on $\mathrm{P} 2 \mathrm{Y} 12$ at randomisation

TIMI=thrombolysis in myocardial infarction. $C A B G=$ coronary artery bypass graft. NSTEMI=non-ST segment elevation myocardial infarction. STEMI=ST segment elevation myocardial infarction.

$\mathrm{PCl}=$ percutaneous coronary intervention. GRACE= Global Registry of Acute Coronary Events. NA=not applicable. 


\begin{tabular}{|c|c|c|c|c|}
\hline & $\begin{array}{l}\text { Aspirin } \\
(n=1518)\end{array}$ & $\begin{array}{l}\text { Rivaroxaban } \\
(n=1519)\end{array}$ & $\mathrm{HR}(95 \% \mathrm{Cl})$ & $\mathrm{p}$ value \\
\hline $\begin{array}{l}\text { Cardiovascular death, } \\
\text { myocardial infarction, stroke, } \\
\text { or definite stent thrombosis }\end{array}$ & $72(5 \%)$ & $76(5 \%)$ & $1.06(0.77-1.46)$ & 0.7316 \\
\hline Cardiovascular death & $17(1 \%)$ & $19(1 \%)$ & $1.12(0.58-2.15)$ & 0.7401 \\
\hline Myocardial infarction & $49(3 \%)$ & $56(4 \%)$ & $1.15(0.78-1.68)$ & 0.4872 \\
\hline Stroke & $12(1 \%)$ & $7(<1 \%)$ & $0.58(0.23-1.48)$ & 0.2506 \\
\hline All-cause death & $23(1 \cdot 5 \%)$ & $22(1 \%)$ & $0.95(0.53-1.71)$ & 0.8771 \\
\hline All stent thrombosis & $16(1 \%)$ & $17(1 \%)$ & $1.06(0.54-2.11)$ & 0.8583 \\
\hline Definite stent thrombosis & $8(1 \%)$ & $11(1 \%)$ & $1.37(0.55-3.42)$ & 0.4917 \\
\hline Probable stent thrombosis & $1(<1 \%)$ & $1(<1 \%)$ & $1.00(0.06-16.00)$ & 0.9997 \\
\hline Possible stent thrombosis & $7(<1 \%)$ & $5(<1 \%)$ & $0.72(0.23-2 \cdot 25)$ & 0.5649 \\
\hline
\end{tabular}

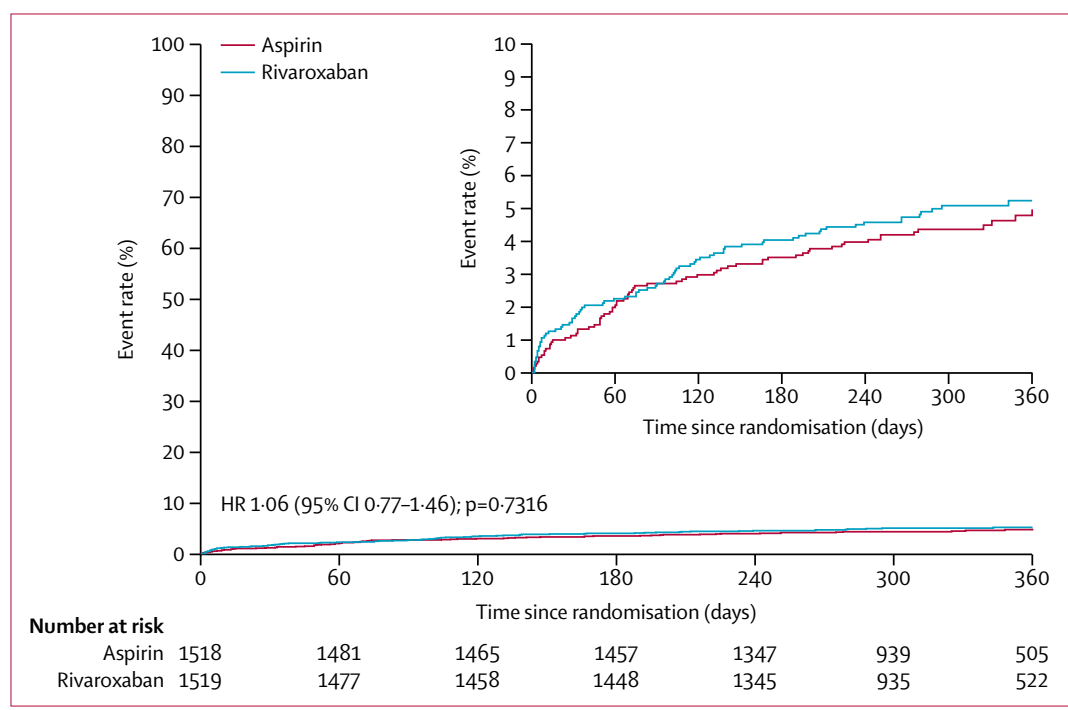

Figure 4: Cardiovascular death, myocardian infarction, stroke, or definite stent thrombosis between treatment groups

$\mathrm{HR}=$ hazard ratio. previous trials assessing triple antithrombotic therapy showed a greater than two-fold increase in bleeding risk, we chose an upper boundary of $2 \cdot 0$ for the confidence interval surrounding the hazard ratio for bleeding, to estimate a sample size that would provide some confidence around this observation. This is distinct from a formal non-inferiority hypothesis testing strategy, which is usually used for phase 3 trials. Our observations suggest that the dual pathway strategy might be non-inferior to that of DAPT in this trial $\left(\mathrm{p}_{\text {non-inferiority }}<0 \cdot 0001\right)$ using the upper bound of $2 \cdot 0$ for the confidence interval, but because this was a phase 2 trial it would require a larger study to confirm.

Notwithstanding the potential mitigation of bleeding risk with the removal of aspirin from the antithrombotic regimen in the post-PCI setting for patients with an indication for oral anticoagulation such as atrial fibrillation, the potential hazard of increased thrombotic risk with this approach (especially early in the post-acute coronary syndromes setting) remains uncertain. Current trials assessing the safety and efficacy of removing aspirin between 30 and 90 days after PCI, with continued use of P2Y12 inhibitor monotherapy thereafter, are enrolling a broad population of patients with PCI. ${ }^{23}$ As such, these trials are not specifically focusing on patients with post-acute coronary syndromes whose risk of recurrent ischaemic events is the highest and potentially most susceptible to optimised antithrombotic therapy regimens. ${ }^{23}$

In the GEMINI-ACS-1 trial, most patients were treated with PCI where there is a risk of stent thrombosis. Studies have noted a substantially higher risk of stent thrombosis when a P2Y12 inhibitor is stopped early after PCI in patients with acute coronary syndromes..$^{24-26}$ In these studies, the risk of stent thrombosis increased more than four-fold when clopidogrel was stopped early after PCI. Less is known about the risk of stent thrombosis among patients who stopped aspirin but continued treatment with P2Y12 inhibitors. Ongoing studies are testing whether P2Y12 inhibitors can be used as monotherapy in the post-PCI setting in the absence of aspirin. $^{27}$ In these trials, aspirin removal is planned 1-3 months after PCI. In the GEMINI-ACS-1 trial, aspirin was substituted with rivaroxaban on average 5 days after the index event and no substantial increase in the risk of stent thrombosis was noted. The upper bound for stent thrombosis observed was $2 \cdot 1$ in this study. This does not preclude a small increased risk of stent thrombosis, but muted effect on stent thrombosis was seen with low-dose rivaroxaban monotherapy in an animal model, and was observed when rivaroxaban was added to aspirin and clopidogrel in the ATLAS-2 trial..$^{15,28-30}$ Although we also noted no apparent differences in the risk of composite ischaemic events by treatment assignment, these are hypothesis-generating findings

In addition to being the largest trial assessing a dual pathway antithrombotic regimen in the post-acute coronary 
syndromes setting, GEMINI-ACS-1 represents the first major clinical experience with low-dose rivaroxaban plus standard-dose ticagrelor. This combination has rarely been studied but the previous finding from the PLATO trial of potential harm with high-dose aspirin together with ticagrelor was a key factor in the decision to use low-dose aspirin (100 mg daily) as the active comparator in GEMINI-ACS-1. ${ }^{31}$ The results of GEMINI-ACS-1 suggest that combining rivaroxaban with ticagrelor, in the absence of aspirin, did not cause a substantially increased risk of clinically significant bleeding compared with aspirin plus ticagrelor. We also did hypothesis-generating multivariate modelling to better ascertain the effect of P2Y12 inhibitor use and the primary bleeding endpoint. We did identify that the use of ticagrelor in this trial was associated with an increased bleeding risk. The higher bleeding rate was associated with regions in which there was a greater use of ticagrelor, but there was no association with the randomised treatment assignment, suggesting that a larger trial testing either ticagrelor or clopidogrel with rivaroxaban would offer a better chance to understand this complex relation between practice pattern and bleeding outcomes. The observations here should be interpreted with caution because of their post-hoc nature and lack of power.

The strongest evidence of the use of aspirin comes from trials done in the 1980s in which aspirin was predominantly used to treat patients in the first 30 days. $^{2}$ In our trial we replaced aspirin with rivaroxaban in the first week after myocardial infarction. In a hypothesisgenerating landmark analysis, we noted a numerically higher but not significant ischaemic event rate in the first 30 days. Although our trial is not large enough to explore this issue in a more meaningful way, it does suggest that a more intense antithrombotic therapy is required in the acute phase of myocardial infarction. Previous studies have suggested more activated platelets in the acute phase of myocardial infarction, ${ }^{32}$ while thrombin generation seems to persist over a longer period in the post-acute coronary syndromes setting. ${ }^{33}$ Although this hypothesis is generated from post-hoc observations that lack power, future studies should try to address this issue. Defining the best intensity of antithrombotic therapies, while patients transition from the acute thrombotic setting to chronic prevention, deserves more work that promises to offer the benefit of reducing both ischaemic and bleeding events in an optimal manner. This complexity is further challenged by the need to develop a trial and regulatory strategy that can discern these important facets.

There are several limitations of the study. It should be recognised that the patients had to be on stable therapy with either clopidogrel or ticagrelor with aspirin for $48 \mathrm{~h}$ before they could be randomly assigned to either aspirin or rivaroxaban. This led to a delay of about 5 days from the index event to randomisation. The study population was relatively homogeneous with few patients being nonwhite, which should be considered when interpreting the findings. Finally, we did not include patients treated with prasugrel because this therapy is restricted to PCI-treated patients only and the intent of our trial was to study the full spectrum of acute coronary syndromes.

In summary, this phase 2 study assessed rivaroxaban in combination with P2Y12 inhibitors approved for use in acute coronary syndromes, and we show that this dual pathway antithrombotic regimen replacing aspirin with low-dose rivaroxaban had a similar risk of clinically significant bleeding. Based upon these findings and the unmet need for improved treatments in the post-acute coronary syndromes setting, further testing of the efficacy and safety of this novel dual pathway antithrombotic regimen in an adequately powered trial should be considered.

\section{Contributors}

EMO, MTR, and CMG were responsible for the conception and design of the work, contributed to the collection and interpretation of the data and drafted the first version of the report. PGS, SKJ, TJP, AP, HM, JS, XS, SH, MT, GM, MCB, DA, HB, MJC, JCN, JHC, SG, RGK, UG, D-WP, $\mathrm{CB}$, and RCW contributed to the data collection, reviewed the data, and contributed to the content and critical revision of the report. EMO, MTR, CMG, FR, and JW had access to the data. FR and JW did the statistical analyses and contributed to the content and critical revision of the report. All authors agreed to submit the report for publication.

\section{Declaration of interests}

EMO has received institutional research grants from Janssen Pharmaceuticals, Daiichi-Sankyo, and Gilead Sciences; consulting fees from Abbott Vascular, Abiomed, AstraZeneca, Bayer, Biotie, Boehringer Ingelheim, Daiichi-Sankyo, Medscape, Merck, St. Jude Medical, Stealth Peptides, and The Medicines Company. MTR has received institutional research grants from Janssen Pharmaceuticals, Eli Lilly, Daiichi-Sankyo, Sanofi-Aventis, Ferring Pharmaceuticals, Myokardia, AstraZeneca, American College of Cardiology, American Heart Association, Familial Hypercholesterolemia Foundation; and consulting fees from Eli Lilly, Daiichi-Sankyo, Myokardia, AstraZeneca, PriMed, Boehringer Ingelheim, Merck, Actelion, Amgen, Novartis, Quest Diagnostics, and Elsevier Publishers. PGS has received consulting fees and non-financial support (member of GEMINI steering committee) from Janssen Pharmaceuticals; and research grants from Sanofi; consulting fees from Amarin, AstraZeneca, Bayer, Boehringer Ingelheim, Daiichi-Sankyo, GlaxoSmithKline, Lilly, Merck-Sharpe-Dohme, Novartis, Pfizer, Roche, Medtronic, Sanofi, Servier, The Medicines Company, CSL Behring, Regeneron, and Bristol-Myers Squibb. SKJ has been a steering committee member (non-financial support) for Janssen

Pharmaceuticals; and has received research grant from AstraZeneca and consulting fees from Bayer. TJP has received institutional research grant from Janssen Pharmaceuticals. JW declares no competing interests. FR has received institutional research grant from Janssen Pharmaceuticals, and AstraZeneca; and consulting fees from AbbVie, Amgen, GlaxoSmithKline, Nabriva, Adverum, and NovoNordisk. AP is an employee of Janssen Pharmaceuticals Research \& Development. HM is an employee of Bayer. JS is an employee of Janssen Pharmaceuticals Research \& Development. XS is an employee of Janssen Pharmaceuticals Research \& Development. MT has received consulting fees from Janssen Pharmaceuticals, Bayer, Celyad, and Servier; and research grants from EU Framework Program VII and the Polish National Center for Research and Development. GM has received institutional research grants from ADIR, Amgen, AstraZeneca, Bayer, Boehringer Ingelheim, Bristol-Myers Squibb, Celladon, Daiichi-Sankyo, Eli Lilly, ICAN, Fédération Française de Cardiologie, Medtronic, MSD, Pfizer, Sanofi-Aventis, and The Medicines Company; and consulting fees from Amgen, AstraZeneca, Bayer, Berlin Chimie AG, Boehringer Ingelheim, Bristol-Myers Squibb, Beth Israel Deaconess Medical, Brigham and Women's Hospital, Cardiovascular Research Foundation, CME Resources, Daiichi-Sankyo, Eli Lilly, Europa, Elsevier, Fondazione 
Anna Maria Sechi per il Cuore, Gilead, Janssen, Lead-Up, Menarini, MSD, Pfizer, Sanofi-Aventis, The Medicines Company, TIMI Study Group, and WebMD. MCB declares no competing interests. DA has received research grants and consulting fees from AstraZeneca, Bayer GlaxoSmithKline, Eli Lilly, Pfizer, and Novartis; and consulting fees from Boehringer Ingelheim, Johnson \& Johnson, Daiichi Sankyo, Boston Scientific, Bristol-Myers Squibb, Menarini, and Sanofi Aventis. HB has received a research grant from AstraZeneca; and consulting fees from Janssen Pharmaceuticals, Abbott, AstraZeneca, BMS-Pfizer, Ferrer, Novartis, and Servier; non-financial support from Sanofi. MJC has received consulting fees from AstraZeneca, Bayer, and Daiichi Sankyo. JCN has received institutional research grants from Janssen Pharmaceuticals, AstraZeneca, Sanofi, Boehringer Ingelheim, and BMS consulting fees from Janssen Pharmaceuticals, AstraZeneca, Sanofi, and Boehringer Ingelheim; and an educational grant from Bayer. JHC has received a research grant from Boehringer Ingelheim; and consulting fees from Bayer and AstraZeneca. SG has received a research grant from Sanofi; and speakers' bureau fees from AstraZeneca. RGK has received speakers' bureau fees from Pfizer, Boehringer Ingelheim, Bayer, and MSD. UG has received consulting fees from Janssen Pharmaceuticals and Bayer. D-WP declares no competing interests. CB has received consulting fees from Bayer. RCW has received research grants and consulting fees from AstraZeneca, Amgen, Bayer, Boehringer Ingelheim, BMS/Pfizer, Janssen, and Edwards LifeSciences. CMG has received research grants from Janssen Pharmaceuticals, Angel Medical Corporation, Bayer, CSL Behring, Ikaria Inc., Johnson \& Johnson, Stealth Peptides, St Jude Medical, and Portola Pharmaceuticals; and consulting fees from Bayer, The Medicines Company, Boston Clinical Research Institute, Cardiovascular Research Foundation, Eli Lilly, Gilead Sciences, Novo Nordisk, Pfizer, WebMD, and UpToDate in Cardiovascular Medicine.

\section{Acknowledgments}

Janssen Research \& Development (Raritan, NJ, USA) and Bayer AG (Wuppertal, Germany) funded the GEMINI-ACS-1 trial and supported this analysis through a grant to Duke University. All analyses were conducted at the Duke Clinical Research Institute (Durham, NC, USA) and the authors had full access to all data. The Duke Clinical Research Institute coordinated the trial, managed the database, and performed the secondary and post-hoc analyses for this manuscript independent of the sponsors. An international executive committee designed the trial and was responsible for oversight of study conduct and reporting of all results and takes responsibility for the accuracy and completeness of data analyses. The authors are fully responsible for the study design, data collection, analysis and interpretation of the data, and writing of the manuscript. The sponsor played no role in the decision to submit the manuscript for publication. Elizabeth Cook, an employee of the Duke Clinical Research Institute, provided editorial support.

\section{References}

1 ISIS-2 (Second International Study of Infarct Survival Collaborative Group. Randomised trial of intravenous streptokinase, oral aspirin, both, or neither among 17,187 cases of suspected acute myocardial infarction: ISIS-2. Lancet 1988; 2: 349-60.

2 Antithrombotic Trialists' (ATT) Collaboration. Aspirin in the primary and secondary prevention of vascular disease: collaborative meta-analysis of individual participant data from randomised trials. Lancet 2009; 373: 1849-60.

3 Yusuf S, Zhao F, Mehta SR, Chrolavicius S, Tognoni G, Fox KK. Effects of clopidogrel in addition to aspirin in patients with acute coronary syndromes without ST-segment elevation. N Engl J Med 2001; 345: 494-502.

4 Wiviott SD, Braunwald E, McCabe $\mathrm{CH}$, et al. Prasugrel versus clopidogrel in patients with acute coronary syndromes. N Engl J Med 2007; 357: 2001-15.

5 Wallentin L, Becker RC, Budaj A, et al. Ticagrelor versus clopidogrel in patients with acute coronary syndromes. N Engl J Med 2009; 361: 1045-57.

6 Amsterdam EA, Wenger NK, Brindis RG, et al. 2014 ACC/AHA guideline for the management of patients with non-ST-elevation acute coronary syndromes: a report of the American College of Cardiology/American Heart Association Task Force on Practice Guidelines. Circulation 2014; 130: e344-426.
7 Roffi M, Patrono C, Collet JP, et al. 2015 ESC Guidelines for the management of acute coronary syndromes in patients presenting without persistent ST-segment elevation. Eur Heart J 2016; 37: 267-315.

8 O'Gara PT, Kushner FG, Ascheim DD, et al. 2013 ACCF/AHA guideline for the management of ST-elevation myocardial infarction: a report of the American College of Cardiology Foundation/American Heart Association Task Force on Practice Guidelines. J Am Coll Cardiol 2013; 61: e78-140.

9 Steg PG, James SK, Atar D, et al. ESC guidelines for the management of acute myocardial infarction in patients presenting with ST-segment elevation. Eur Heart J 2012; 33: 2569-619.

10 Roe MT, Ohman EM. A new era in secondary prevention after acute coronary syndrome. N Engl J Med 2012; 366: 85-87.

11 Mega JL, Braunwald E, Wiviott SD, et al. Rivaroxaban in patients with a recent acute coronary syndrome. $N$ Engl J Med 2012; 366: 9-19.

12 Alexander JH, Lopes RD, James S, et al. Apixaban with antiplatelet therapy after acute coronary syndrome. N Engl J Med 2011; 365: 699-708.

13 Dewilde WJM, Oirbans T, Verheugt FWA, et al. Use of clopidogrel with or without aspirin in patients taking oral anticoagulant therapy and undergoing percutaneous coronary intervention: an open-label, randomised, controlled trial. Lancet 2013; 381: 1107-15.

14 Gibson CM, Mehran R, Bode C, et al. Prevention of bleeding in patients with atrial fibrillation undergoing PCI. N Engl J Med 2016; 375: 2423-34.

15 Becker EM, Perzborn E, Klipp A, et al. Effects of rivaroxban, acetylsalicylic acid and clopidogrel as monotherapy and in combination in a porcine model of stent thrombosis. $J$ Thromb Haemost 2012; 10: 2470-80.

16 Perzborn E, Heitmeier S, Laux V. Effects of rivaroxaban on platelet activation and platelet-coagulation pathway interaction: In vitro and in vivo studies. J Cardiovasc Pharmacol Ther 2015; 20: $554-62$.

17 Povsic TJ, Roe MT, Ohman EM, et al. A randomized trial to compare the safety of rivaroxaban versus aspirin in addition to either clopidogrel or ticagrelor in acute coronary syndrome: the design of the GEMINI-ACS-1 phase II study. Am Heart J 2016; 174: $120-28$

18 Husted S, Emanuelsson H, Heptinstall S, Sandset PM, Wickens M Peters G. Pharmacodynamics, pharmacokinetics, and safety of the oral reversible P2Y12 antagonist AZD6140 with aspirin in patients with atherosclerosis: a double-blind comparison to clopidogrel with aspirin. Eur Heart J 2006; 27: 1038-47.

19 Mega JL, Braunwald E, Mohanavelu S, et al. Rivaroxaban versus placebo in patients with acute coronary syndromes (ATLAS ACS-TIMI 46): a randomised, double-blind, phase II trial. Lancet 2009; 374: 29-38.

20 Mehran R, Rao SV, Bhatt DL, et al. Standardized bleeding definitions for cardiovascular clinical trials: a consensus report from the Bleeding Academic Research Consortium. Circulation 2011; 123: 2736-47.

21 Thygesen K, Alpert JS, Jaffe AS, et al. Third universal definition of myocardial infarction. Circulation 2012; 126: 2020-35.

22 Hicks KA, Tcheng JE, Bozkurt B, et al. 2014 ACC/AHA key data elements and definitions for cardiovascular endpoint events in clinical trials: a report of the American College of Cardiology/American Heart Association task force on clinical data standards (Writing Committee to Develop Cardiovascular Endpoints Data Standards). J Am Coll Cardiol 2015; 66: 403-69.

23 Welsh RC, Roe MT, Steg PG, et al. A critical reappraisal of aspirin for secondary prevention in patients with ischemic heart disease. Am Heart J 2016; 181: 92-100.

24 Schulz S, Schuster T, Mehilli J, et al. Stent thrombosis after drug-eluting stent implantation: incidence, timing, and relation to discontinuation of clopidogrel therapy over a 4-year period. Eur Heart J 2009; 30: 2714-21.

25 Iakovou I, Schmidt T, Bonizzoni E, et al. Incidence, predictors, and outcome of thrombosis after successful implantation of drug-eluting stents. JAMA 2005; 293: 2126-30.

26 Mehran R, Baber U, Steg PG, et al. Cessation of dual antiplatelet treatment and cardiac events after percutaneous coronary intervention (PARIS): 2 year results from a prospective observational study. Lancet 2013; 382: 1714-22. 
27 Gargiulo G, Windecker S, Vranckx P, et al. A critical appraisal of aspirin in secondary prevention: is less more? Circulation 2016; 134: 1881-906.

28 Gibson CM, Chakrabarti AK, Mega J, et al. Reduction of stent thrombosis in patients with acute coronary syndromes treated with rivaroxaban in ATLAS-ACS TIMI 51. J Am Coll Cardiol 2013; 62: 286-90.

29 Steg PG, Harrington RA, Emanuelson H, et al. Stent thrombosis with ticagrelor versus clopidogrel in patients with acute coronary syndromes: an analysis from the prospective, randomized PLATO trial. Circulation 2013; 128: 1055-65.

30 Wiviott SD, Braunwald E, McCabe $\mathrm{CH}$, et al. Intensive oral antiplatelet therapy for reduction of ischaemic events including stent thrombosis in patients with acute coronary syndromes treated with percutaneous coronary intervention and stenting in the TRITON-TIMI 38 trial: a subanalysis of a randomized trial. Lancet 2008; 371: 1353-63.
31 Mahaffey KW, Wojdyla DM, Carroll K, et al. Ticagrelor compared with clopidogrel by geographic region in the Platelet Inhibition and Patient Outcomes (PLATO) trial. Circulation 2011; 124: 544-54.

32 Ohman EM, Harrington RA, Lincoff AM, Kitt MM, Kleiman NS Tcheng JE. Early clinical experience with integrelin, an inhibitor of the platelet glycoprotein IIb/IIIa integrin receptor. Eur Heart J 1995 16S: 50-55.

33 Merlini PA, Ardissino D, Rosenberg RD, et al. In vivo thrombin generation and activity during and after intravenous infusion of heparin or recombinant hirudin in patients with unstable angina pectoris. Arterioscler Thromb Vasc Biol 2000; 20: 2162-66. 